Aceptado: Julio 2018

\title{
El pago por méritos en los derechos audiovisuales del fútbol español: ¿debemos primar el espectáculo (goles) o la clasificación?
}

\section{The merit payment for broadcasting rights of spanish football: should we prioritize the show (goals) or the league table position?}

\author{
Jaime Pinilla*, Miguel Ángel Negrín y Christian González-Martel \\ Departamento de Métodos Cuantitativos en Economía y Gestión. Universidad de Las Palmas de Gran Canaria (España).
}

\begin{abstract}
Resumen: En los últimos años el consumo de eventos futbolísticos a través de los medios audiovisuales está superando los mercados nacionales para globalizarse. La sostenibilidad económica de los clubes de fútbol depende cada vez más de la difusión masiva en medios audiovisuales y de su capacidad para mantenerse como un producto atractivo, competitivo y socialmente relevante. En la actualidad en muchas ligas europeas, entre ellas la española, el lugar que ocupa un equipo al final del campeonato repercute en los ingresos por televisión, en los cinco años siguientes, recibidos por la venta conjunta de los derechos de audiovisuales, es el conocido como pago por mérito. Sin embargo, la clasificación no tiene por qué estar relacionada con el espectáculo que ofrece un equipo en el campo, y por tanto con las posibilidades comerciales de sus partidos en países donde el fútbol está buscando su lugar como deporte espectáculo.

Palabras clave: Demanda televisiva de fútbol, derechos de retransmisión, pago por mérito, número de goles.
\end{abstract}

Abstract: In recent years the consumption of football events through audiovisual media has transcend national borders to globalize. The economic sustainability of the football clubs increasingly depends on media diffusion and their capacity to remain as an attractive, competitive and socially relevant product. Currently, in many European football leagues, including the Spanish "La Liga", the club's final position within the league table has a direct impact on the next five years' revenues from TV broadcasting as a result of the joint selling of football broadcasting rights. This is commonly known as merit payment. However, the league table position does not have to be related to the quality of the match or the level of entertainment offered by the football team in the field, and therefore with the commercial possibilities of their matches in countries where football is not yet conceived as a sport of entertainment.

Key words: Television demand for football, broadcasting rights, merit payment, goal scoring.

\section{Introducción}

Cuando un nuevo aficionado se sienta frente al televisor para ver un partido de fútbol lo primero que busca es entretenerse. Antes de jurar fidelidad a ningún equipo, el espectador busca disfrutar del espectáculo, ver un juego elegante y vistoso, ver goles (Simmons, 1996). Nos referimos a espectadores por ejemplo de China o Estados Unidos, espectadores de países donde el fútbol está buscando su lugar como deporte espectáculo, espectadores a los cuales un partido entre equipos que proponen una estrategia ultradefensiva, aparcar el autobús delante de la portería, los lleva a cambiar de canal o apagar el televisor. A la espera de los datos del Real Madrid-FC Barcelona de la temporada 2017-2018, el partido con más televidentes en el país asiático, más de 26 millones (datos de CSM-KantarSport Asia), corresponde al R. Madrid-Granada de la temporada 2014-2015. Resultado final 9-1, 7-0 a la media hora de partido.

En los últimos años, la literatura ha mostrado la relación entre mejores audiencias televisivas y el juego ofensivo con mayor número de goles. Alavy, Gaskell, Leach, y Szymans-

\footnotetext{
Dirección para correspondencia [Correspondence address]: Jaime Pinilla. Universidad de Las Palmas de Gran Canaria (España). E-mail: jaime.pinilla@ulpgc.es
}

ki (2010) estudiaron las audiencias minuto a minuto, de los partidos de la Premier League, y encontraron que los espectadores por lo general prefieren los partidos con marcadores abiertos y con un número elevado de goles. Similares resultados se obtuvieron analizando los datos de audiencias de la Serie A en Italia (Caruso, Addesa y Di Domizio, 2017). Sólo el hincha más fervoroso está dispuesto a sacrificar el espectáculo por un empate o victoria por la mínima que beneficie a su equipo (Buraimo y Simmons, 2009).

Sin embargo, contrariamente a lo esperado, el juego de ataque y un mayor número de goles por partido no está reconocido en el reparto de los derechos de televisión. En muchas ligas europeas parte de los ingresos derivados de la comercialización de los contenidos audiovisuales se distribuye entre los equipos de acuerdo con su clasificación en las últimas temporadas, es el llamado pago por méritos. En la medida en que los puntos en la clasificación no siempre reflejan el espectáculo que da un equipo (Andreff y Raballand, 2011), esta propuesta de pago por mérito podría incluso ir en contra de las posibilidades de comercialización del deporte rey en mercados emergentes. Con el propósito de ocupar un buen puesto en la clasificación, los equipos buscan sumar 3 puntos en cada encuentro, y para ello sólo es necesario marcar un gol 
más que el equipo contrario, y esto se consigue tanto con un 1-0 como con un 5-2.

En la primera división de la liga de fútbol española, la mitad de todos los ingresos obtenidos por la explotación centralizada de derechos audiovisuales se distribuye a partes iguales entre los 20 clubes de la competición. La cantidad restante se divide a su vez en dos partes, la primera atendiendo a los resultados deportivos obtenidos, pago por mérito, y la segunda atendiendo a la implantación social del cada club, valorada esta última a partir de la recaudación en abonos y taquilla media y la generación de recursos de comercialización en las retransmisiones televisivas nacionales.

El pago por mérito supone por tanto el $25 \%$ del dinero repartido a los clubs. Este pago se realiza de acuerdo con un baremo vinculado a la clasificación de las últimas 5 últimas temporadas, ponderándose la clasificación obtenida en la última un $35 \%$, en la penúltima un $20 \%$ y un $15 \%$ cada una de la tres anteriores. La cuantía asignada a cada temporada se distribuye entre los participantes del siguiente modo: el primer clasificado recibe el $17 \%$, el segundo un $15 \%$, el tercero un $13 \%$, y así sucesivamente hasta el vigésimo que recibe un 0,25\% (ver Real Decreto 5/2015).

En el último reparto de ingresos por televisión de 2017 en la primera división, 311,66 millones de euros correspondieron al pago por méritos. De acuerdo con la cantidad anterior, pasar del puesto octavo al undécimo, se traduce en 3,4 millones menos en las próximas cinco temporadas, retroceder un peldańo del puesto quinto al sexto cuesta 6,8 millones, etc.

El objetivo principal de este trabajo es mostrar las diferencias en el reparto del pago por mérito para los equipos de la primera división de la liga española según el criterio actual, la clasificación final alcanzada en las últimas 5 temporadas, y una clasificación alternativa de acuerdo con el número de goles esperado por partido en cada una de estas temporadas, proxy del nivel de espectáculo. Como objetivo secundario se comparan las 5 mayores ligas europeas (Inglaterra, España, Italia, Alemania y Francia) respecto de la distribución del número de goles esperado por partido.

\section{Método}

\section{Instrumentos}

La calidad entendida como espectáculo de un partido de fútbol resulta muy difícil de modelizar debido a la baja puntuación (número de goles) en este deporte. Quién se erige como equipo ganador e incluso el marcador final casi nunca coincide con la percepción sobre la calidad del juego de cada contrincante (Borland y Macdonald, 2003).

Como alternativa al resultado final del encuentro, número de goles, se propone utilizar el número de goles esperado para cada equipo como proxy del potencial ofensivo. El número de goles esperado está corregido por las condiciones bajo las cuales se consigue el gol, la situación del marcador, el minuto de partido, el número de remates, la distancia, el ángulo, habilidad del jugador, etc., y sus orígenes se relacionan con el trabajo pionero de Ensum, Pollard y Taylor (2003).

Existen varias propuestas, los dos indicadores más conocidos son el xG "expected golas" de optasports (Green, 2012) y los recientes Offensive y Defensive Soccer Power Index propuestos por el equipo de FiveThirtyEight liderado por Nate Silver. El Offensive Soccer Power Index (OSPI) es el indicador utilizado en nuestro análisis.

\section{Muestra}

Información desagregada de los resultados de todos los partidos, en las últimas 17 temporadas, de las 5 ligas de fútbol más importantes de Europa. Especial atención a los resultados de la primera división española en las 5 temporadas utilizadas para el reparto de los derechos televisivos en 2017/2018. Valores del OSPI para 11 equipos de la primera división española que se han mantenido en la primera categoría entre la temporada 2012/2013 y 2016/2017.

\section{Procedimiento}

Ordenación de los equipos de acuerdo con el criterio actual, clasificación, y el propuesto, indicador OSPI. Cuantificación de las diferencias en la cuantía correspondiente al pago por mérito en la temporada 2017/2018 atendiendo a una u otra clasificación.

\section{Resultados}

\section{Comparativa internacional}

La figura 1 presenta la evolución, entre las temporadas 2000/2001 y 2016/2017, del número medio de goles por partido en las 5 competiciones de fútbol más importantes de Europa: Bundesliga Alemania, Ligue 1 Francia, Serie A Italia y La Liga Espańa. La Bundesliga es la que presenta inicialmente mayor media de goles por partido, superada a partir de la temporada 2012/2013 por la Premier y La Liga. La Ligue 1 es la competición con menor número medio de goles por partido. Resulta interesante resaltar como a partir de la temporada 2008/2009 la cola de la distribución del número medio de goles se alarga hacia la izquierda, destacándose unos pocos equipos con un enorme potencial goleador, es el caso de la liga española.

En consonancia con la figura anterior, la Ligue 1 es la competición con menor nivel de asistencia a los estadios, así como audiencias televisivas más bajas (Andreff y Raballand, 2011), por tanto, un producto de difícil comercialización internacional. 


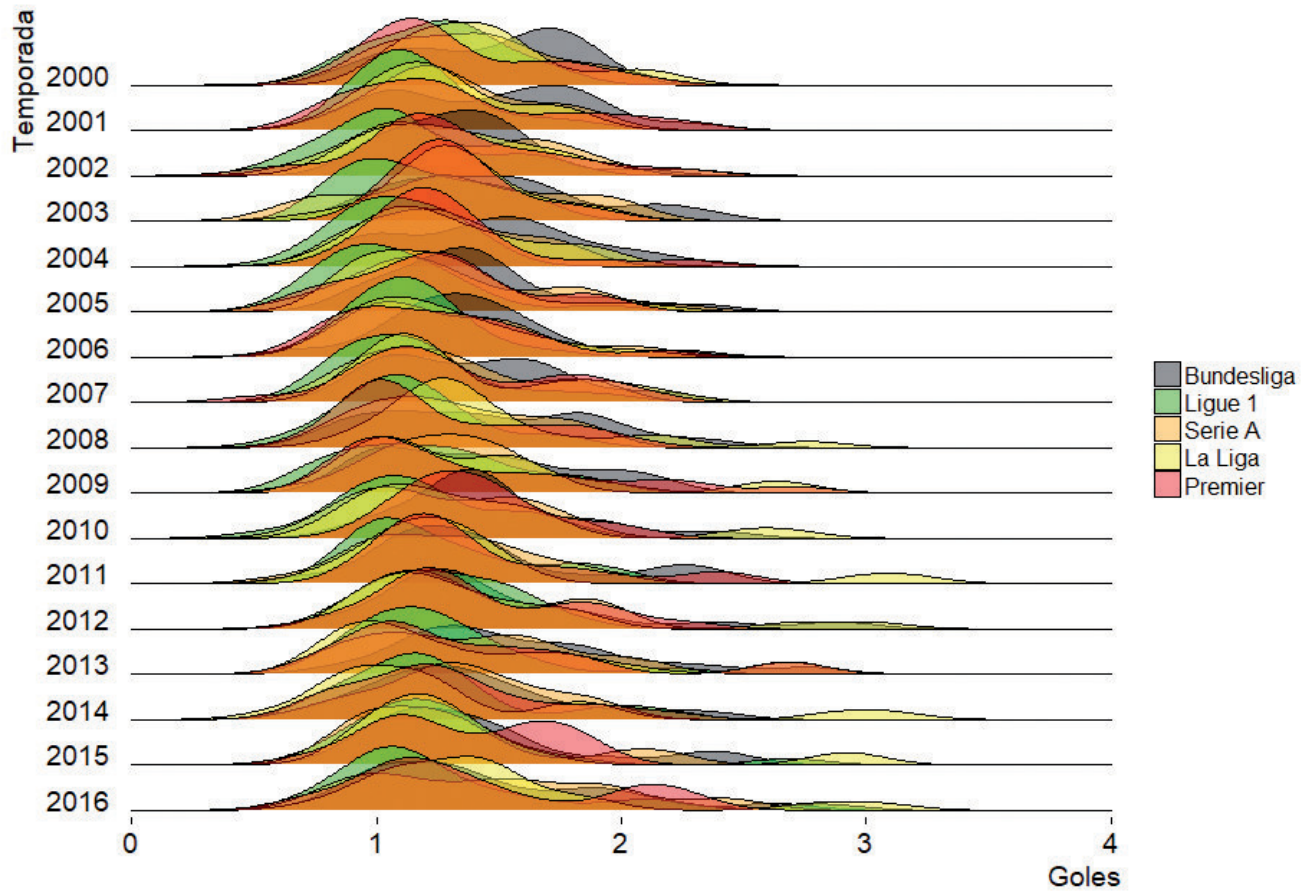

Figura 1. Evolución del número medio de goles por partido: Temporada 2000/01 -2016/17.

A continuación, la figura 2 ordena los equipos de cuatro ligas europeas (dejamos fuera la Ligue 1 francesa) teniendo en cuenta los indicadores de potencial ofensivo y defensivo propuestos por Nate Silver. Como potencial ofensivo se entiende el número medio esperado de goles por partido de cada equipo, mientras que el potencial defensivo equivale al número medio de goles que el equipo espera encajar en cada partido. Las líneas rojas discontinuas se cortan en el valor medio y dividen la figura en cuatro cuadrantes. El primer cuadrante recogería a los equipos con mayor potencial ofensivo y que a su vez encajan menor número de goles.

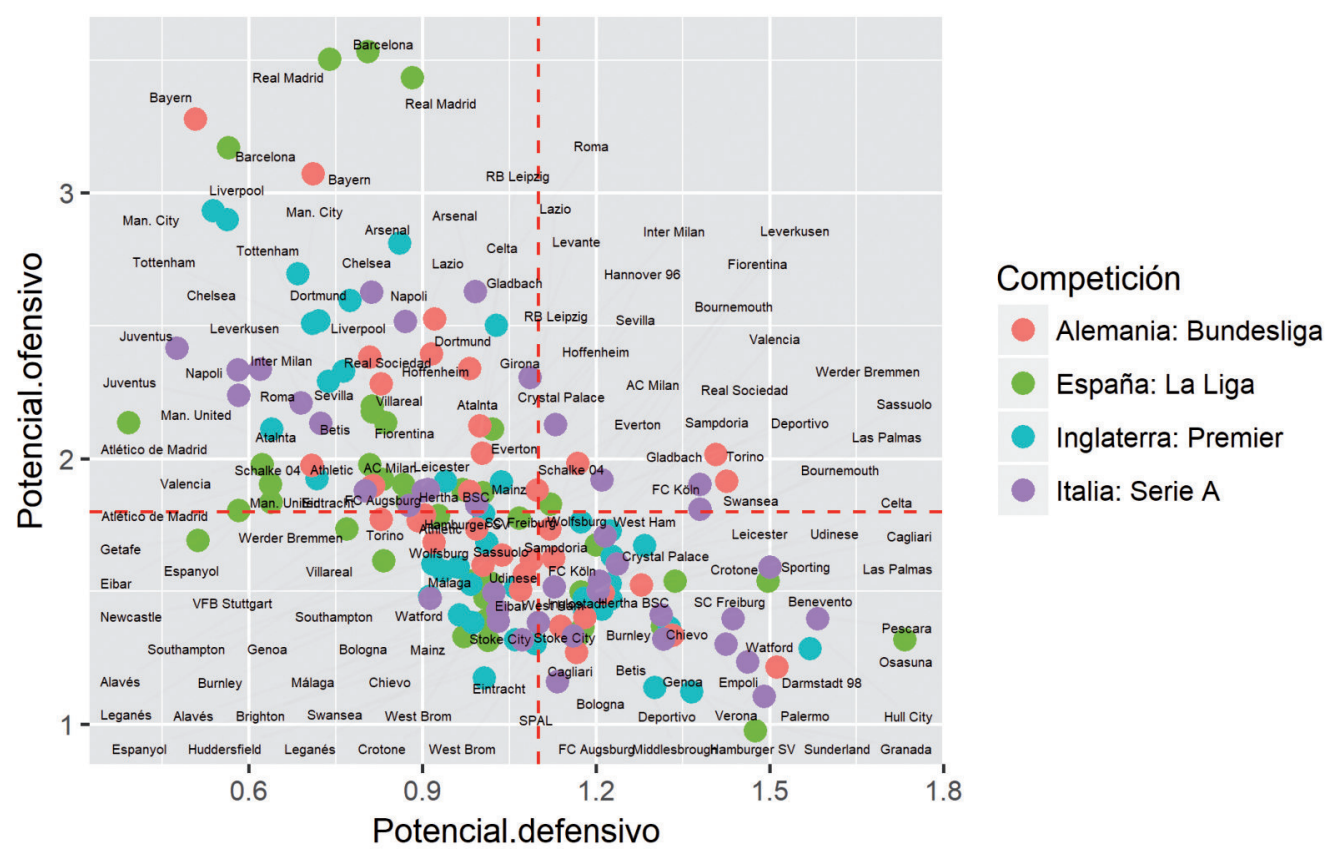

Figura 2. Clasificación de los equipos de las 4 mejores ligas europeas en relación a su potencial ofensivo y defensivo: temporadas 2016/2017 y 2017/2018. 
El Real Madrdid y el F.C. Barcelona son junto al Bayer de Munich los equipos con mayor potencial ofensivo en europa. Por competiciones, la Bubdesliga con 7 de 18 equipos en el primer cuadrante supera al resto de ligas europeas en número de goles esperado.

\section{Comparativa nacional}

Atendiendo sólo al potecial ofensivo, la figura 3 presenta la ordenación de 11 equipos de la primera división española que se han mantenido en dicha categoría entre las temporadas 2012/2013 y 2016/2017. Real Madrid y FC Barcelona dominan claramente al resto de equipos, a continuación se alternan equipos como la Real Sociedad, Atlético de Madrid, Sevilla y Valencia.

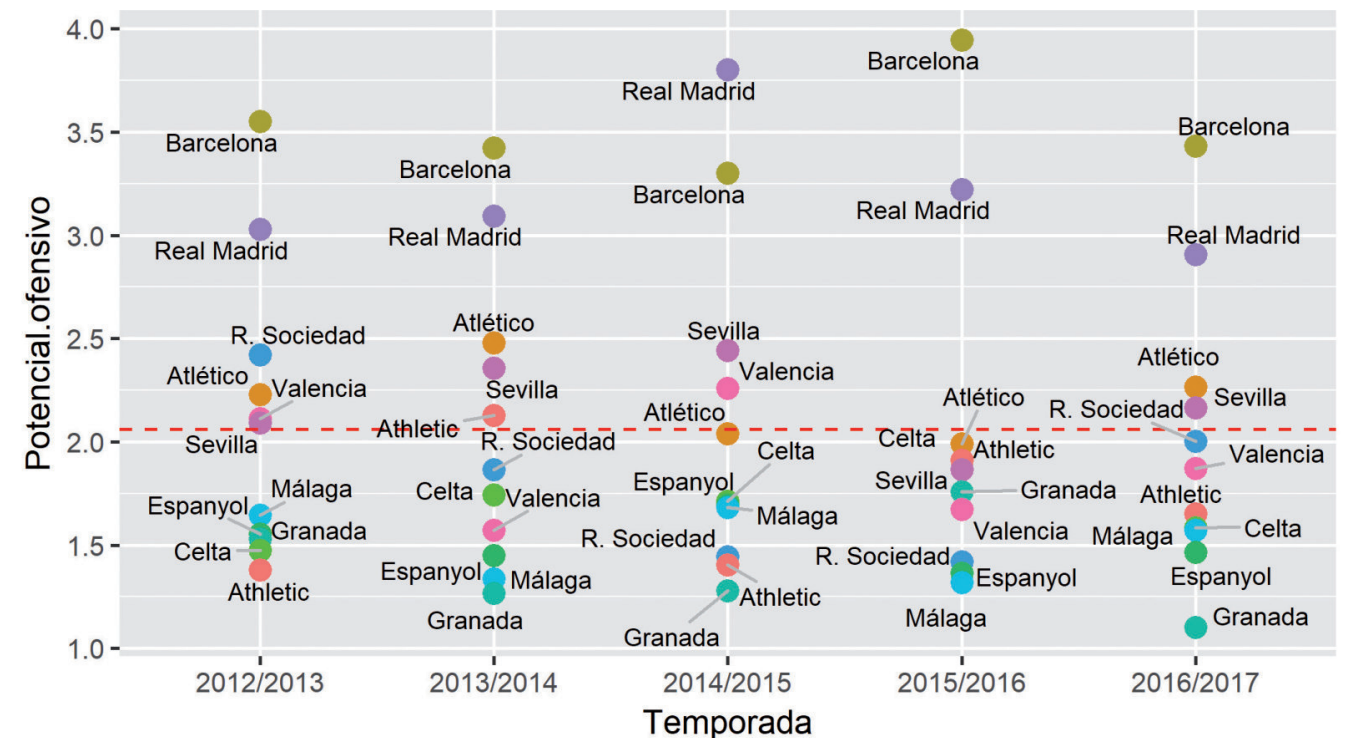

Figura 3. Ranking respecto al potencial ofensivo en La Liga: últimas 5 temporadas (11 equipos).

La ordenación mostrada en la figura 3 hubiera tenido claras consecuencias en el reparto de las cantidades del pago por mérito, ver tabla 1. Atlético de Madrid y Málaga son los equipos más beneficiados por el actual criterio de reparto, clasificación, mientras que el Sevilla es el más penalizado con casi
7,5 millones de euros menos. El caso del Atlético de Madrid no sorprende, desde que en 2012 Simenone cogió el banquillo, el resultado 1-0 se produce en el $32 \%$ de sus encuentros (Diario As, 24 de abril de 2017).

Tabla 1. Diferencias en el pago por mérito (millones de euros) de 2016/2017 si se tiene en cuenta el potencial ofensivo del equipo frente a la clasificación al final de temporada.

\begin{tabular}{lccc}
\hline Equipo & $\begin{array}{c}\text { Reparto actual } \\
\text { (clasificación) }\end{array}$ & $\begin{array}{c}\text { Reparto propuesto } \\
\text { (potencial ofensivo) }\end{array}$ & Diferencia \\
\hline Athletic de Bilbao & 19,59 & 19,67 & 0,08 \\
Atlético de Madrid & 42,39 & 37,71 & $-4,67$ \\
FC Barcelona & 49,87 & 52,05 & 2,18 \\
Celta & 10,05 & 14,03 & 3,97 \\
Espanyol & 8,10 & 6,31 & $-1,79$ \\
Granada & 2,77 & 4,32 & 1,56 \\
Málaga & 10,75 & 6,58 & $-4,17$ \\
Real Sociedad & 18,04 & 18,04 & 0 \\
Real Madrid & 48,00 & 47,69 & $-0,31$ \\
Sevilla & 22,13 & 29,61 & 7,48 \\
Valencia & 14,84 & 16,21 & 1,36 \\
\hline
\end{tabular}




\section{Discusión}

Cuando los derechos de retransmisión de la Premier League se vendieron por primera vez en 1983, estos alcanzaron los 2,5 millones de libras esterlinas. Recientemente, los canales Sky Sports y BT Sports acaban de desembolsar más de 4,4 billones, miles de millones, de libras para la retransmisión, en exclusividad, en el mercado nacional entre 2016 y 2022.

A pesar de las abultadas cifras anteriores, los directivos de la Premier saben que el verdadero potencial de crecimiento se encuentra fuera de Inglaterra. La Premier League es la competición que mejor se ha expandido en países donde no era habitual la presencia del fútbol en los contenidos televisivos, además de la clara ventaja que supone el idioma y ser los primeros en emitir, la flexibilidad de horarios ha sido clave. Sin embargo, gran parte del éxito se atribuye también al valor intrínseco del producto, un fútbol rápido y con goles (Andreff y Raballand, 2011).

Nuestra propuesta sugiere reconocer el mérito de cada equipo en relación con la calidad de su juego, potencial ofensivo. En países donde el fútbol todavía no es un deporte con muchos seguidores, la principal objeción es que el fútbol es aburrido, con marcadores cortos, a diferencia por ejemplo del fútbol americano o el baloncesto. No tiene sentido por tanto condicionar parte de los ingresos de los equipos por derechos televisivos sólo a la clasificación, pues supone un incentivo a las tácticas defensivas que llevan a marcadores con escaso número de goles (Raballand, Cianferani, y Marteau, 2008).

Sin embargo, evaluar el potencial espectáculo no es una tarea fácil. Un equipo puede vencer 4-0 a su rival haciendo sólo 4 disparos a puerta en todo el encuentro. Para mitigar esta circunstancia se necesitan nuevas métricas que estimen mejor la calidad del juego subyacente. En los últimos años, la analítica deportiva en general y del fútbol en particular, ha evolucionado rápidamente (Hughes y Franks, 2005; Tenga, Wheeler y Lyons, 2010; Seippel, 2018). La cada vez mayor disponibilidad de datos nos abre la puerta para intentar evaluar desde la eficiencia goleadora (Villa y Lozano, 2016) hasta el estilo atacante y defensivo de cada equipo (Fernandez-Navarro, Fradua, Zubillaga, Ford y McRobert, 2016).

\section{Conclusiones}

En los últimos años, las grandes ligas de fútbol se han convertido en productoras de contenido audiovisual. La demanda televisiva del fútbol es en la actualidad la mayor fuente de ingresos de las ligas, por consecuencia, si queremos premiar el mérito conviene reconocer aquello que favorece la expansión del mercado, el juego vistoso y con marcadores abultados.

Nuestros resultados para la primera división española muestran que los puntos en la clasificación no siempre coinciden con el espectáculo que da un equipo, la propuesta actual de pago por mérito parece por tanto ir en contra de las posibilidades de comercialización del fútbol en mercados emergentes.

La utilización del número de goles esperado para cada equipo como proxy del potencial ofensivo, parece reflejar mejor el espectáculo ofrecido, pero no es un indicador validado por lo que es una limitación de nuestro estudio. La ausencia en la literatura de índices o indicadores sobre el potencial espectáculo que proporciona cada equipo es un campo interesante para futuras investigaciones. En la actualidad todos los encuentros se monitorizan con mucho detalle por lo que no resulta difícil recopilar información sobre ocasiones, posesión, y múltiples aspectos del juego con los que construir indicadores de estilo los cuales relacionar con las correspondientes audiencias televisivas.

\section{Referencias}

1. Alavy K., Gaskell, A., Leach, S. \& Szymanski S. (2010). On the Edge of Your Seat: Demand for Football on Television and the Uncertainty of Outcome Hypothesis". International Journal of Sport Finance, 5 (2),7595.

2. Andreff, W., \& Raballand, G. (2011). Is European football's future to become a boring game? In W. Andreff (Ed.), Contemporary issues in sports economics. Participation and professional team sports. Cheltenham, UK and Northampton, MA, USA: Edward Elgar, pp.131-167.

3. BOE núm. 104, de 1 de mayo de 2015. Real Decreto-ley 5/2015, de 30 de abril, de medidas urgentes en relación con la comercialización de los derechos de explotación de contenidos audiovisuales de las competiciones de fútbol profesional.

4. Borland, J., \& Macdonald, R.D, (2003). Demand for Sport. Oxford Review of Economic Policy, 19 (4),478-502. doi: 10.1093/oxrep/19.4.478

5. Buraimo, B., \& Simmons, R. (2009). A tale of two audiences: Spectators, television viewers and outcome uncertainty in Spanish football. Journal of Economics and Business, 61, 326-338. doi: 10.1016/j. jeconbus.2008.10.002
6. Caruso, R., Addesa, F., \& Di Domizio M., (2017). The Determinants of the TV Demand for Soccer Empirical Evidence on Italian Serie A for the Period 2008-2015. Journal of Sports Economics, Disponible online desde 12 Jul 2017. doi: 10.1177/1527002517717298

7. Diario deportivo As (2017, April 24). El Atleti, abonado este cruso al 1-0 con gol de Griezmann. Retrieved from https://as.com/futbol/2017/04/23/primera/1492959832_724987.html

8. Ensum, J., Pollard, R., \& Taylor S. (2003). Applications of logistic regression to shots at goal in association football. Paper presented at $5^{\text {th }}$ World Congress on Science and Football, Lisbon, April.

9. Fernandez-Navarro, J., Fraduab, L., Zubillagac, A., Forda, P.R. \& McRoberta, A.P. (2016). Attacking and defensive styles of play in soccer: analysis of Spanish and English elite teams. Journal of Sport Sciences 34 (24), 2195-2204. doi: 10.1080/02640414.2016.1169309

10. FiveThirtyEight. (2010, August 10). What's New In Our 2017-18 Club Soccer Predictions [Blog post]. Retrieved from https://fivethirtyeight. com/features/whats-new-in-our-2017-18-club-soccer-predictions/

11. Green, S. (2012). Assessing the Performance of Premier Leauge Goals- 
corers. OptaPro Blog [Blog post]. Retrieved from http://www.optasportspro.com/about/optapro-blog/posts/2012/blog

12. Hughes, M., \& Franks, I. (2005). Analysis of passing sequences, shots and goals in soccer. Journal of Sports Sciences, 23 (5), 509-514. doi: $10.1080 / 02640410410001716779$

13. Kantar Espańa Insights (2015) La hora $\mathrm{H}$ y el día D del fútbol espańol en Asia. Retrieved from https://es.kantar.com/media/deporte/2015/ abril-2015-datos-audiencia-partido-m\%C3\%A1s-visto-en-chinaen-la-temporada-2014-2015/

14. Seippel, Ø. (2018). Attacking beautifully or defending efficiently? A sociological analysis of the prevalence and effect of football strategies. Soc- cer and Society, 9 (2), 185-204. doi: 10.1080/14660970.2015.1133411 15. Simmons, R. (1996) The Demand for English League Football: A ClubLevel Analysis. Applied Economics, 28, 139-55.

16. Tenga, A., Holme, I., Ronglan, L.T. \& Bahr, R. (2010). Effect of playing tactics on goal scoring in Norwegian professional soccer. Journal of Sports Sciences, 28 (3), 237-244. doi: 10.1080/02640410903502774

17. Raballand, G., Cianferani, S., \& Marteau, J.F. (2008). Quel avenir pour le football? Objectif $0-0$. Paris: L'Harmattan.

18. Villa, G. \& Lozano, S. (2016). Assessing the scoring efficiency of a football match. European Journal of Operational Research, 255 (2), 559-569. doi:10.1016/j.ejor.2016.05.024 